\title{
The Galactic Center excess brought down-to-earth.
}

\section{Daniele Gaggero}

SISSA, via Bonomea 265, 34136 Trieste (Italy) \& INFN, Sezione di Trieste, via Valerio 2, 34127

Trieste (Italy).

E-mail: daniele.gaggero@sissa.it

\section{Marco Taoso}

Institut de Physique Théorique, CNRS URA2306 \& CEA-Saclay, 91191 Gif-sur-Yvette (France).

E-mail: Marco.Taoso@cea.fr

\section{Piero Ullio}

SISSA, via Bonomea 265, 34136 Trieste (Italy) \& INFN, Sezione di Trieste, via Valerio 2, 34127

Trieste (Italy).

E-mail: Piero.Ullio@sissa.it

\section{Alfredo Urbano*}

SISSA, via Bonomea 265, 34136 Trieste (Italy) \& INFN, Sezione di Trieste, via Valerio 2, 34127

Trieste (Italy).

E-mail: alfredo.urbano@sissa.it

\section{Mauro Valli}

SISSA, via Bonomea 265, 34136 Trieste (Italy) \& INFN, Sezione di Trieste, via Valerio 2, 34127

Trieste (Italy).

E-mail: Mauro.Valliesissa.it

There is evidence for a spherical-symmetric diffuse gamma-ray signal peaked in the Galactic Center (GC) region and extended up to several degrees off the GC. We critically review some relevant points regarding the derivation of this signal by means of the template-fitting method. In particular we turn our attention to the Inverse Compton (IC) template usually adopted for the background emission, and we show that it does not provide a realistic description of the complex GC environment. Driven by the evidence of a large gas density in the inner kpc of the Galaxy correlated with an impressive Supernova rate ( 2 order of magnitude larger than the Galactic average), and therefore with ongoing CR acceleration, we show that the template-fitting algorithm does not show any clear evidence of the aforementioned excess if a realistic modification in the diffuse CR source term is implemented.

The 34th International Cosmic Ray Conference,

30 July- 6 August, 2015

The Hague, The Netherlands

\footnotetext{
*Speaker.
} 


\section{The GeV gamma-ray excess}

Several authors have reported since 2009 the detection of a gamma-ray signal from the inner few degrees around the Galactic Center (GC) [1, 2, 3, 4]. Its spectrum and morphology are claimed to be compatible with those expected from annihilating dark matter (DM) particles [5]. Many theoretical models have been proposed on the model-building side $[6,7,8]$. Given that the emission peaks at relatively low energies (few $\mathrm{GeV}$ ), and that the DM explanation implies an annihilation cross section of the order of the typical one for thermal Weakly Interacting Massive Particles (WIMPs), the DM scenario seems to be quite appealing, being also compatible with well motivated theories Beyond the Standard Model. Moreover, the DM hypothesis is not ruled out by the constraints from other observations [9, 10], in particular anti-protons measurements and gamma-ray observations of dwarf galaxies. Nevertheless, several interesting astrophysical interpretations of the signal were proposed as well: the presence a population of unresolved millisecond pulsars [11, 12], a bursting star-forming activity in the past near the GC [13], or nonthermal bremsstrahlung produced by a population of electrons interacting with neutral gas in molecular clouds [14, 15]. In [16] we presented an alternative astrophysical interpretation of the GC excess. The starting point of our analysis is based on a critical examination of the gamma-ray background in the GC region.

\section{An excess relative to what?}

The detection of an excess, by definition, should be based on a solid and comprehensive knowledge of the background, and the GC excess does not deviate from this rule. The evidence of the GC excess is obtained adopting the so-called template fitting algorithm. The rationale of this technique is that the current models aimed at simulating the gamma-ray diffuse emission from the Galaxy are not accurate enough to reproduce the morphology of the signal with the desired accuracy. The method consists in allowing the templates of the different components (mainly the Inverse Compton (IC), $\pi^{0}+$ bremsstrahlung and Extra-Galactic background template) to freely fluctuate in a Poisson likelihood fit. The relative normalizations of the template providing the best fit to the data are retained. This exercise is performed independently for each energy bin in the desired range. Within this framework, the investigation of a DM related excess is done adding a DM-like template to the process, and verifying if this extra ingredient is used by the fitting algorithm or not. This point is well explained in the left panel of fig. 1 where we show the typical outcome of the template fitting method (see caption for details). We focus on a region of interest (ROI) centered in the GC with $|l|<20^{\circ}, 2^{\circ} \leqslant|b| \leqslant 20^{\circ}$ in Galactic coordinates. The DM component (here modeled according to a generalized Navarro-Frenk-White distribution with $\gamma=1.26$ ) clearly emerges from the fitting procedure. This is even more clear from fig. 2 where we show, the residuals (i.e. the difference data - model) without (left panel) and with (central panel) the inclusion of the DM template.

\section{Towards a realistic astrophysical interpretation}

The templates used for the fit in fig. 1 are taken from the "Model A" in [17]. This specific choice does not alter significantly the result. The authors of [17], in fact, proved that the DM component enlightened by the template fitting procedure explained before is stable against a large set of 

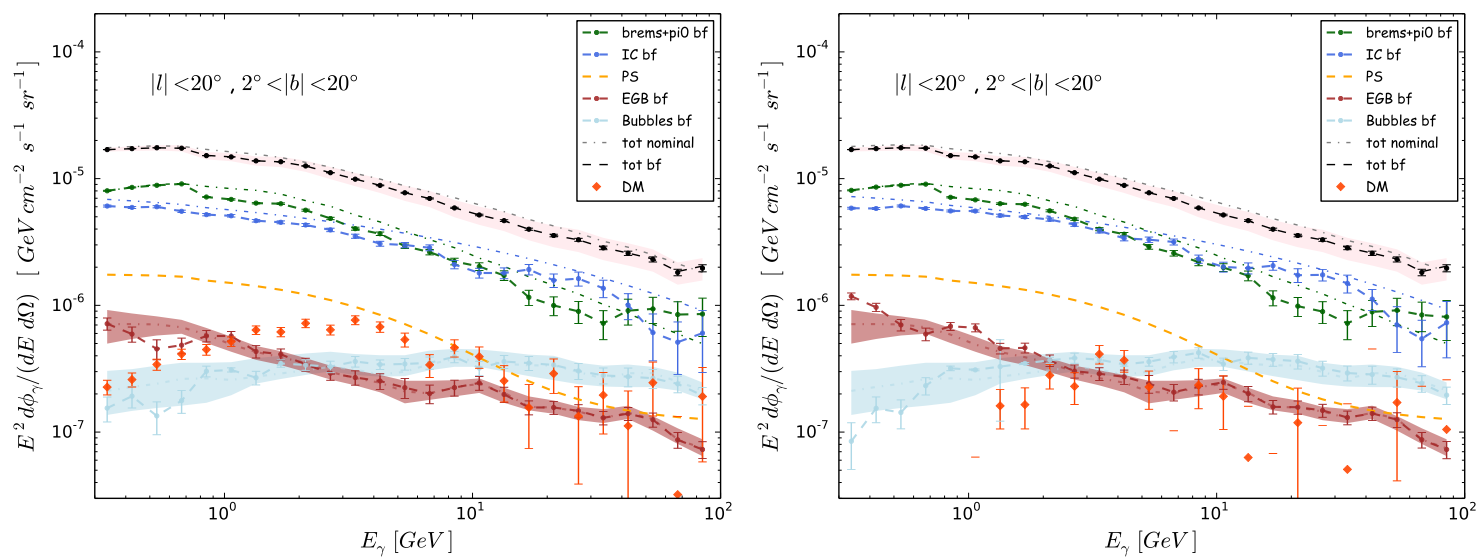

Figure 1: Spectrum of the various contributions to the total gamma-ray flux, pre- and post-template fitting, compared to the Fermi-LAT data. Left panel. Standard astrophysical background model. Right panel. Spike background model.
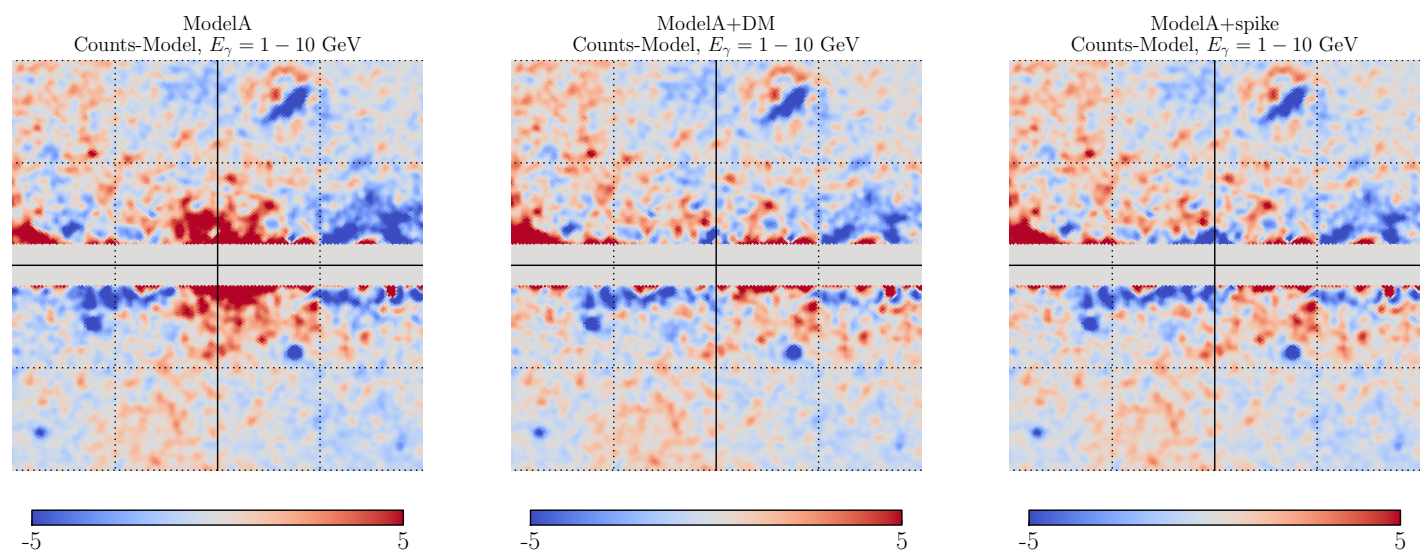

Figure 2: Template fitting residuals without (left panel) and with (central panel) the inclusion of the DM template in the presence of a standard astrophysical background model. In the right panel we show the residuals for the spike background model (without DM template).

models adopted for the Galactic diffuse emission. However, all the models discussed in [17] rely on assumptions on the cosmic-ray (CR) density based on standard CR propagation models originally built with the purpose of fitting the local observables, completely unaffected by the details of the central regions. For this reason, they are not adequate at all for this kind of study since they do not include a realistic description of the CR source distribution and CR diffusion in the inner regions of the Galaxy. The purpose of [16] is to show that within a more realistic description of CR production in the inner part of the Galaxy, the excess is not present anymore. To be more precise, the new ingredient we add is a steady-state source located in the GC with a narrow spatial extension (few hundred pc). Many observations based on robust astrophysical measurements support our picture. We know that the GC is likely to harbour significant star formation and a large rate of Supernova explosions compared to the average value in the Galaxy: according to [18], the star formation rate (SFR) in the GC region is $\sim 1 \%$ of the SFR in the Galaxy, and this rate is is roughly a factor of 250 higher than the mean rate in the Galaxy. This should be the consequence of a large reservoir of 
molecular gas filling the inner part of the Galactic bulge (dubbed Central Molecular Zone [19] and extending up to $\sim 200 \div 300$ pc far from the GC) and of the very peculiar physical properties of this environment: the insterstellar medium appears significantly hotter, more turbulent, and more highly magnetized. An extraordinary evidence supporting this estimate regarding the SFR comes from the infrared observations - performed with the Hubble Space Telescope—of some extremely dense stellar clusters in the inner $50 \mathrm{pc}$ (the Central, Arches, and Quintuplet clusters) rich of young, very hot stars that are many times larger and more massive than the Sun (see e.g. [20, 21] and references therein). Moreover, many isolated Wolf-Rayet Stars and O Supergiants were observed in the inner $100 \mathrm{pc}$ [22]. The Supernova explosions connected with this relevant star formation activity are expected to accelerate a large amount of CRs. Although this contribution has no impact at all on the local observations, the consequences on the gamma-ray emission modeling are very important, as we will show in the rest of the paper.

\section{Strategy, results and discussion}

The starting point of the analysis is a physical model for the CR distribution in the Galaxy obtained with DRAGON $[23,24]$. This numerical code is designed to simulate all processes related to Galactic CR transport (in particular: diffusion, reacceleration, convection, energy losses, spallation) for all CR species, from heavy nuclei to protons, antiprotons, and leptons. DRAGON can work in both 2D and 3D mode, and in both cases it is possible to implement anisotropic diffusion. The code includes the nuclear cross section database and interstellar radiation field model taken from the latest public version of Galprop ${ }^{1}$. We then perform a line-of-sight integral and obtain the gamma-ray skymaps from $\simeq 0.3 \mathrm{GeV}$ to $\simeq 300 \mathrm{GeV}$ using GammaSky [25]. This package computes the gamma-ray emission due to: 1) decay of neutral pions produced by collision of CRs with the interstellar gas; 2) IC scattering of CR leptons on the diffuse radiation field; 3) Bremsstrahlung emission due to CR leptons interacting with the interstellar gas. We adopt, as a reference, the propagation model labelled as "ModelA" in [17]. Starting from this model, we add a steady-state source of CRs in the central region and we study the interplay between the emitted CRs and an environment. The source in the center (hereafter, "spike") is modeled as a Gaussian:

$$
Q_{\text {spike }}=Q_{0} \exp \left[\frac{x^{2}+y^{2}+z^{2}}{\sigma^{2}}\right]
$$

In the following, we will express the normalization in terms of $\mathscr{N}$, i.e. the ratio (in percent) between the volume integral of the spike and the volume integral of the conventional source term. This provides an easier comparison with observational constraints on the CR injection term in the center (correlated to the Star formation rate, or the Supernova explosion rate). The most relevant parameters describing the new ingredients under consideration are he spatial extension $\sigma$ and the normalization $\mathscr{N}$ of the spike. In the following, we present a reference case corresponding to the simplest version of the model, characterized by isotropic diffusion and by an optimal choice for the extension and normalization of the spike. We show that this scenario provides a satisfactory fit of all the observables that we are considering. We refer the interested reader to [16] for a more

\footnotetext{
${ }^{1}$ http: //galprop.stanford.edu/
} 
quantitative description of the template fitting procedure, data analysis, and for possible variations from the reference case discussed here.
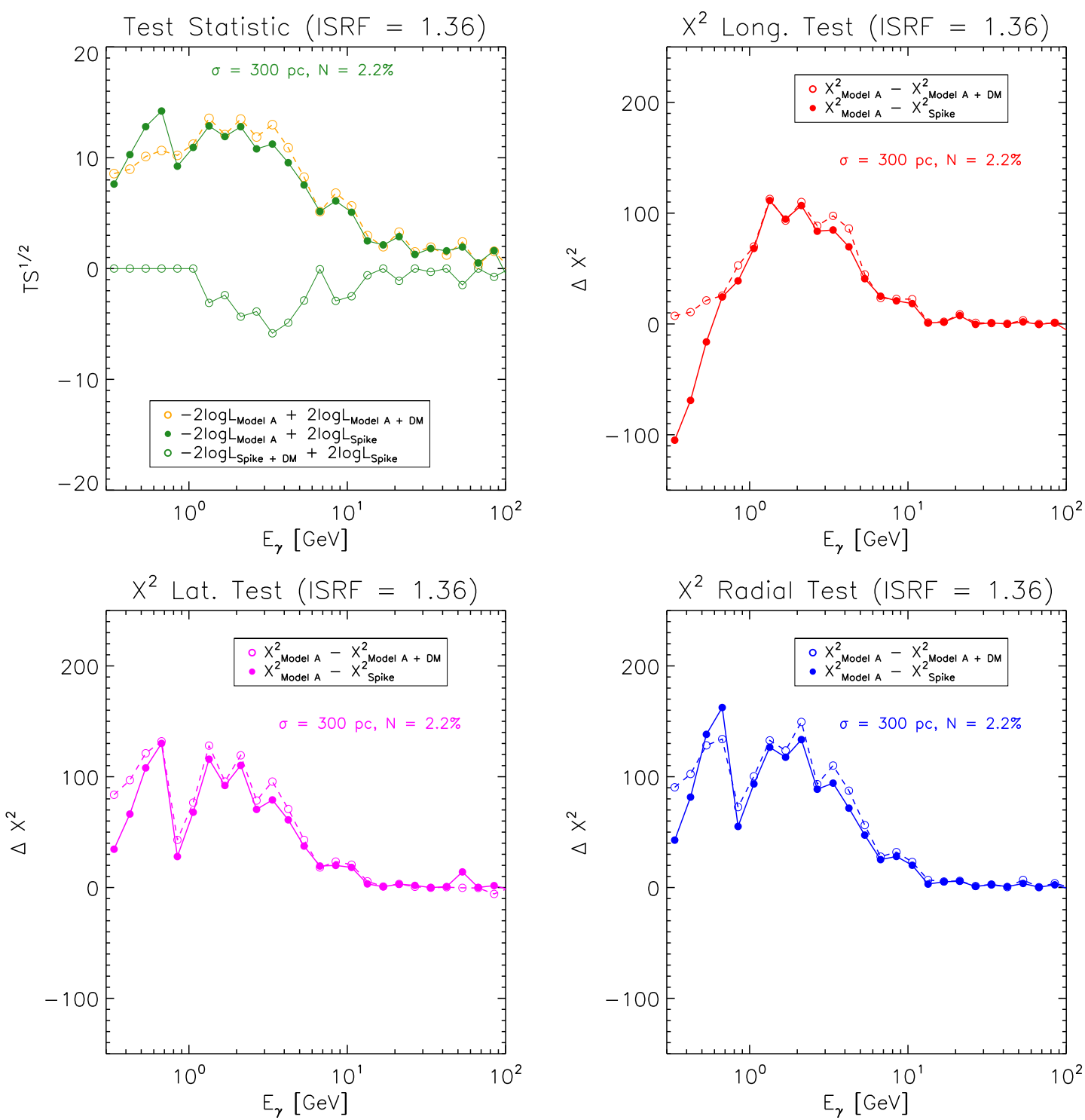

Figure 3: Top left panel. We compare the test-statistic $(\mathrm{TS}=-2 \Delta \log \mathscr{L})$ of the models we consider; a positive difference between two models means that the first model performs better. Yellow filled circles: $-2 \log \mathscr{L}_{\text {Model A }}+2 \log \mathscr{L}_{\text {Model A+DM. }}$. Green filled circles: $-2 \log \mathscr{L}_{\text {Model A }}+2 \log \mathscr{L}_{\text {Spike. }}$. Green empty circles: $-2 \log \mathscr{L}_{\text {Spike+DM }}+2 \log \mathscr{L}_{\text {Spike }}$. Top right panel. We compare the $\chi^{2}$ of the longitude profiles for the same models. Filled circles: $\chi_{\text {Model A }}^{2}-\chi_{\text {Spike }}^{2}$; empty circles: $\chi_{\text {Model A }}^{2}-\chi_{\text {Model A+DM. }}^{2}$ Bottom panels. The same as the top right panel, for latitude and radial profiles.

Our analysis is based on the comparison between the reference model defined before, and the case in which we add to Model A the DM template. The inclusion of a DM template in the fitting procedure in addition to standard astrophysical background models-like the Model A adopted in this analysis - provided the most striking evidence supporting the DM interpretation of the GC excess. In [5], the addition of the DM template dramatically improved the fit up to an overwhelming 
high level of statistical significance. For this reason, it is mandatory to confront the performances of the spike model with those of the DM template, and we organize our analysis in three subsequent steps.

1) Energy spectra. For the reference case we take $\sigma=300 \mathrm{pc}$. We fix the normalization following a very simple selection criterium based on two objective requirements: the value of $\mathscr{N}$ must be chosen in such a way to absorb the majority of the GC excess, and the value of $\mathscr{N}$ cannot be too large, since the SFR in the GC cannot exceed few percent of the total rate in the Galaxy. In the right panel of fig. 1 we show the best fit obtained including the DM template for a normalization $\mathscr{N}=2.2 \%$ : the contribution of the DM template is small and, most importantly, gives rise to a featureless spectrum. This is even more clear from the right panel of fig. 2: undeniably, most of the GC excess was absorbed by the presence of the spike.

2) Test Statistic. In order to scrutiny the performance of our model, let us now consider the upper left panel of fig. 3 where we compare, for each energy bin, the Test Statistic (TS) of the models we are studying. The TS is defined by TS $=-2 \Delta \log \mathscr{L}$, where $\mathscr{L}$ is the likelihood function defining the template fitting algorithm (cfr. [16] for details). First, we show the improvement in the TS obtained adding the DM template to the Model A. More precisely, the yellow dashed line with open circles in fig. 3 represents the square root of the difference $-2 \log \mathscr{L}_{\text {Model A }}+2 \log \mathscr{L}_{\text {Model A+DM }}$ (hereafter, $\Delta \mathrm{TS}$ ). In terms of energy spectra, the case Model A + DM corresponds to the one shown in the left panel of fig. 1. The plot clearly shows the improvement in the fit due to the presence of the DM template. If taken at face value, it corresponds to a statistical preference of about $15 \sigma$ at the position of the peak. Of course we remark that this value should be taken with a huge pinch of salt, since - in addition to the extremely small statistical errors - data are plagued by unavoidable systematics errors not included in the likelihood fit. However, it is indisputable that including the DM template greatly improves the fit; therefore, it is crucial to compare this result with the performance of our spike. The green solid line with filled circles represents the improvement in the fit (quantified by $\Delta \mathrm{TS}$ ) obtained considering our reference spike model with respect to the Model A, The plot clearly shows that our scenario-without any DM contribution-performs better than the starting Model A, and gives a result comparable (even at the level of statistical preference) with the DM case. For completeness, we also show the TS for the combination of the spike and the DM template. The green solid line with open circles represents the corresponding $\Delta \mathrm{TS}$, so that negative values indicate a statistical preference for the addition of the DM template. In terms of energy spectra, this situation corresponds to the right panel of fig. 1. The TS plot shows that the addition of the DM template slightly improves the fit in the energy window $E_{\gamma}=1-10 \mathrm{GeV}$; however-as already noticed - this improvement cannot be interpreted as the evidence of a DM contribution since the majority of the excess was absorbed by the presence of the spike.

3) Longitude, latitude and radial profiles. In order to compare models and data, we analyze the gamma-ray profiles investigating three complementary directions. We compute the latitude (longitude) profiles averaging on $|l| \leqslant 5^{\circ}\left(2^{\circ} \leqslant|b| \leqslant 5^{\circ}\right)$, and we use $1^{\circ}$ bins. The Galactic disk is always masked for $|b| \leqslant 2^{\circ}$. Radial profiles are defined in terms of the angular distance $r \equiv$ $\sqrt{l^{2}+b^{2}}$. We show our results for the longitudinal direction in the upper-right panel of fig. 3 . Latitude and radial profiles are shown, respectively, in the lower-left and lower-right panel of fig. 3. First, we compute - for each direction, and for each energy bin - the observed profiles of gammaray data. Second, using these data and the corresponding statistical errors, we compute the value 
of the $\chi^{2}$ function for each one of the analyzed models, using the best-fit coefficients obtained from the template fitting procedure explained in the previous section. We repeat the analysis for the Model A, the Model A + DM and our reference model. In the three aforementioned panels of fig 3 we superimpose the differences $\Delta \chi_{\mathrm{DM}}^{2} \equiv \chi_{\text {Model A }}^{2}-\chi_{\text {Model A+DM }}^{2}$ (empty circles) and $\Delta \chi_{\text {Spike }}^{2} \equiv$ $\chi_{\text {Model A }}^{2}-\chi_{\text {Spike }}^{2}$ (filled circles). For each line, positive values indicate an improvement in the fit-compared to Model A-obtained including, respectively, the DM template and the spike. In each panel, the relative position of the two lines, $\Delta \chi_{\mathrm{DM}}^{2}$ versus $\Delta \chi_{\text {Spike }}^{2}$, indicate which one of the two ingredients, DM or spike, exhibits a better fit. On a general ground, we will explore the three profiles separating two different energy ranges. On the one hand, the energy region $E_{\gamma}=1-10 \mathrm{GeV}$ (mid-energy region hereafter), where the DM template gives the most pronounced contribution; on the other one, the low-energy tail of the spectrum, $E_{\gamma}=0.3-1 \mathrm{GeV}$. In the mid-energy regionand for all the three analyzed directions - the spike gives a comparable good fit if compared with the DM template, since the role of the DM contribution is entirely played by the IC emission of the spike. As far as the low-energy region is concerned, iwe observe that, in the first two energy bins, the DM template produces a slightly better fit if compared with the spike. This is particularly true for the latitude and longitude profiles. The origin of the discrepancy is clear: while the DM contribution is suppressed at low energy (see fig. 1, left panel), the steady-state emission of the spike does not feature this energy dependence (at least in the simplified model studied in [16]), slightly overshooting the data. Having said that, it is undeniable that this apparently large difference ( $\chi_{\text {Spike }}^{2}-\chi_{\text {Model } \mathrm{A}+\mathrm{DM}}^{2} \sim 100$ in the longitude profiles) is heavily affected by the fact that we included in the computation of the $\chi^{2} \mathrm{~s}$ only statistical errors. A better understanding of the systematic error-estimated to be $5 \%$ at $E_{\gamma}=562 \mathrm{MeV}$, as quoted in [26]—will presumably change the rules of the comparison, washing out, at least partially, the discrepancy.

To sum up, the analysis of the energy spectra, TS, and profiles shows that the presence of the spike in our reference model depicts a viable astrophysical scenario potentially able to fully explain the GC excess.

\section{Conclusions}

We investigated the presence of a GC excess, as reported in many papers, adopting a modified CR source term in the inner Galaxy. We implemented the template-fitting machinery described in [17] and - as a starting point - we adopted the propagation setup dubbed Model A presented in those papers. The computations were performed with the numerical packages DRAGON and Gammasky. Our scenario includes a steady-state CR source (spike) in the GC region, modeled as a Gaussian, whose presence is suggested by several astrophysical observations. We showed that, when the spike is present, the template-fitting algorithm does not adopt the DM template anymore. Very remarkably, from the likelihood point of view, our spike scenario, without any DM, performs better than the background model + DM scenario. A residual discrepancy between model and data is present at very low energy, where the algorithm is less reliable due to the fact that systematic errors are not included, while the statistical ones become negligible.

\section{References}

[1] V. Vitale et al. [Fermi/LAT Collaboration], arXiv:0912.3828 [astro-ph.HE]. 
[2] L. Goodenough and D. Hooper, arXiv:0910.2998 [hep-ph].

[3] D. Hooper and T. R. Slatyer, Phys. Dark Univ. 2 (2013) 118 [arXiv:1302.6589 [astro-ph.HE]].

[4] C. Gordon and O. Macias, Phys. Rev. D 88 (2013) 083521 [arXiv:1306.5725 [astro-ph.HE]].

[5] T. Daylan, D. P. Finkbeiner, D. Hooper, T. Linden, S. K. N. Portillo, N. L. Rodd and T. R. Slatyer, arXiv:1402.6703 [astro-ph.HE]

[6] W.-C. Huang, A. Urbano and W. Xue, JCAP 1404 (2014) 020 [arXiv:1310.7609 [hep-ph]].

[7] A. Alves, S. Profumo, F. S. Queiroz and W. Shepherd, Phys. Rev. D 90, no. 11, 115003 (2014) [arXiv:1403.5027 [hep-ph]].

[8] A. Berlin, D. Hooper and S. D. McDermott, Phys. Rev. D 89, no. 11, 115022 (2014) [arXiv:1404.0022 [hep-ph]].

[9] M. Cirelli, D. Gaggero, G. Giesen, M. Taoso and A. Urbano, JCAP 1412 (2014) 12, 045 [arXiv:1407.2173 [hep-ph]].

[10] M. Ackermann et al. [Fermi-LAT Collaboration], arXiv:1503.02641 [astro-ph.HE].

[11] K. N. Abazajian, JCAP 1103 (2011) 010 [arXiv:1011.4275 [astro-ph.HE]].

[12] J. Petrovic, P. D. Serpico and G. Zaharijas, arXiv:1405.7928 [astro-ph.HE].

[13] E. Carlson and S. Profumo, arXiv:1405.7685 [astro-ph.HE].

[14] F. Yusef-Zadeh, J. W. Hewitt, M. Wardle, V. Tatischeff, D. A. Roberts, W. Cotton, H. Uchiyama and M. Nobukawa et al., Astrophys. J. 762, 33 (2013) [arXiv:1206.6882 [astro-ph.HE]].

[15] O. Macias and C. Gordon, Phys. Rev. D 89, no. 6, 063515 (2014) [arXiv:1312.6671 [astro-ph.HE]].

[16] D. Gaggero, M. Taoso, P. Ullio, A. Urbano, M. Valli, to appear.

[17] F. Calore, I. Cholis, C. Weniger, Journal of Cosmology and Astroparticle Physics, Issue 03, article id. 038, pp. $(20$

[18] Donald F. Figer, R. Michael Rich, Sungsoo S. Kim, Mark Morris, Eugene Serabyn, The Astrophysical Journal, Volume 601, Issue 1, pp. 319-339 (2004)

[19] K. FerriÃíre, W. Gillard, P. Jean, Astronomy and Astrophysics, Volume 467, Issue 2, May IV 2007 , pp.611-627 (2007)

[20] D. F. Figer, F. Najarro, D. Gilmore, M. Morris, S. S. Kim, E. Serabyn, I. S. McLean and A. M. Gilbert et al., Astrophys. J. 581, 258 (2002) [astro-ph/0208145].

[21] D. F. Figer, arXiv:0803.1619 [astro-ph].

[22] J. Mauerhan, A. Cotera, H. Dong, M. Morris, D. Wang, S. Stolovy and C. Lang, Astrophys. J. 725, 188 (2010) [arXiv:1009.2769 [astro-ph.SR]].

[23] C. Evoli, D. Gaggero, D. Grasso and L. Maccione, JCAP 0810 (2008) 018 [arXiv:0807.4730 [astro-ph]].

[24] D. Gaggero, L. Maccione, G. Di Bernardo, C. Evoli and D. Grasso, Phys. Rev. Lett. 111 (2013) 2, 021102 [arXiv:1304.6718 [astro-ph.HE]].

[25] G. Di Bernardo, C. Evoli, D. Gaggero, D. Grasso and L. Maccione, JCAP 1303, 036 (2013) [arXiv:1210.4546 [astro-ph.HE]].

[26] [Fermi-LAT Collaboration], Astrophys. J. 750 (2012) 3 [arXiv:1202.4039 [astro-ph.HE]]. 